\title{
MOL2ALL-VIDEOJUEGO PARA EL APRENDIZAJE DE LA ESTEQUIOMETRÍA: IDENTIFICACIÓN DE CARACTERÍSTICAS DEL JUEGO Y PERFIL DEL JUGADOR
}

\section{Mol2all-videogame for the stoichiometry learning: identification of the charateristics game and player's profile}

\author{
Víctor Hugo Medina García ${ }^{1}$ Laura Marcela Rincón Pérez², Jorge Enrique Otálora Luna \\ ${ }^{1}$ Universidad Distrital Francisco José de Caldas, Grupo de investigación GICODE, Colombia. ${ }^{2}$ Universidad \\ Pedagógica y Tecnológica de Colombia, Grupo de Investigación SIMILES, Colombia. ${ }^{3}$ Universidad Pedagógica y

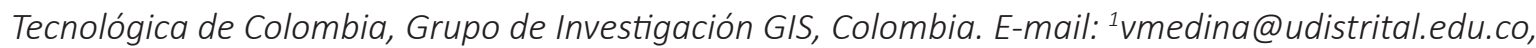 \\ 2laura.rincon08@uptc.edu.co, 3jorge.otalora@uptc.edu.co
}

(Recibido mayo 22 de 2021 y aceptado junio 15 de 2021)

\section{Resumen}

Para gran parte de los estudiantes de educación media aprender estequiometría es complejo porque presentan deficiencias de lógica matemática y manejo de proporciones. Esta situación desmotiva al estudiante durante su proceso de aprendizaje lo que agudiza el problema de comprensión de dicha temática. Se una investigación que dará como resultado la implementación de un videojuego serio que motive el aprendizaje de los cálculos estequiométricos. El alcance del presente artículo es la presentación del estudio realizado para determinar los perfiles de los estudiantes en rol de jugadores, y las características que deberá tener del videojuego que se denominará Mol2All. Para lograrlo, se usó una investigación cuantitativa, con enfoque sintético y diseño cuasi experimental. Se concluye que el perfil del estudiante como jugador está acorde con la metodología de Richard Bartle, además, el estudio arroja que la población que será beneficiaria del videojuego es amplia, sin importar su estrato socioeconómico, su zona de residencia, su género o si la institución educativa es de carácter público o privado. También se determinó que se deberán emplear tecnologías livianas adaptables a todo tipo de dispositivo electrónico en la construcción de Mol2All.

Palabras clave: videojuego serio, estequiometría, enseñanza de la química, aprendizaje de la estequiometría.

\begin{abstract}
For a large part of high school students learning stoichiometry is complex because they have deficiencies in mathematical logic and handling of proportions. This situation demotivates the student during their learning process, which exacerbates the problem of understanding this topic. This study proposes an investigation that will result in the implementation of a serious video game which motivates the learning of stoichiometric calculations. The scope of this article is the presentation of the study carried out to determine the profiles of the students in the role of players, and the characteristics of the video game that will be called Mol2All should have. In order to achieve it, this study used quantitative research, with a synthetic approach and a quasi-experimental design. It concluded that the profile of the student as a player is in accordance with Richard Bartle's methodology, in addition, the study shows that the population that will benefit from the video game is wide, regardless of their socioeconomic status, their area of residence, gender or whether the educational institution is public or private. It was also determined that is important to use lightweight technologies adaptable to all types of electronic devices to be used in the construction of Mol2All.
\end{abstract}

Key words: serious videogame, stoichiometry, chemistry teaching, stoichiometry learning. 


\section{INTRODUCCIÓN}

En el marco del proceso de enseñanza - aprendizaje de la estequiometría, se propone el uso de una herramienta didáctica de apoyo, mediada por un videojuego serio, que motive la comprensión de dicha temática entre los estudiantes de educación media. En la revisión bibliográfica llevada a cabo para determinar cuáles son las dificultades prevalentes en el aprendizaje de los cálculos químicos. Según Obando, Bridges y Galagovsky et al., gran parte de los estudiantes de secundaria presentan falencias en: los razonamientos lógico-matemáticos, interpretaciones, proporciones, entre otras; por lo tanto, tienden a desmotivarse durante el proceso de aprendizaje de la estequiometría [1], [2] y [3].

Por otra parte, Martínez, M. y De Longhi, A., elaboraron una categorización de las dificultades de la lectocomprensión de los problemas estequiométricos de lápiz y papel dificultades detectadas a partir del estudio efectuado por Martínez en 2011-. Los resultados obtenidos les permitió concluir que, las dificultades de lectocomprensión de estos estudiantes no se relacionan únicamente con la habilidad lectora, sino que tienen causas diversas, como las relacionadas con el texto, con la química como disciplina y con los procesos de resolución que exigen los enunciados de los problemas estequiométricos [4].

En cuanto a las estrategias didácticas actuales para abordar la temática de estequiometría, se tienen las basadas en: la Enseñanza para la Comprensión (EPC), el Aprendizaje Basado en Proyectos (ABP), así como aquellas que incluyen: el uso de analogías, integración de TIC e inserción de juego en la secuencia didáctica. La estrategia EPC tuvo mayor éxito en el aprendizaje de la estequiometría por encima del ABP porque: permite la división de temas en módulos más sencillos, implica menor autonomía del estudiante y su curva de aprendizaje es más efectiva, debido a que dista en menor medida al modelo tradicional [5].

Las analogías permiten el uso de un lenguaje simbólico desde modelos que recrean la cotidianidad de los estudiantes y les facilita transponer los conceptos desde el contexto macroscópico al nanoscópico; de esta manera, se propicia la apropiación del conocimiento, siendo el estudiante parte activa en su proceso de aprendizaje de la estequiometría [6].

Adicionalmente, se ha evidenciado que la inserción de las analogías permite la diversificación de la estrategia con la que se presenta la estequiometría, produciendo un efecto motivador, ya que relaciona la cotidianidad y los estudiantes lo valoran de manera positiva [7] y [8]

Existen experiencias en las que se incluyeron las TIC para guiar cursos de estequiometria como las de Obando y Mojica. En la primera, se destaca la motivación que mostraron los estudiantes, así como la posibilidad del seguimiento personalizado que pudo realizar el docente durante el proceso de aprendizaje [1]. La segunda, describe el fortalecimiento de experiencias significativas en el aprendizaje del estudiante, mediante cuatro elementos en la generación de conocimiento: diálogo, reflexión, discusión y consenso [9]. Además, recomiendan la elaboración de un aula virtual que contenga situaciones cotidianas para generar un aprendizaje significativo y que el estudiante adquiera un papel activo en su aprendizaje.

En cuanto a la inclusión de un juego en el proceso de enseñanza-aprendizaje de la estequiometría, existe un estudio que lo aborda como herramienta motivadora dentro de una estrategia didáctica, la cual fue aceptada y aprobada por el $88.9 \%$ de los estudiantes objeto de estudio. A nivel actitudinal, los estudiantes afirmaron que se sintieron más motivados e interesados en el tema, fomentaron el trabajo en equipo y desarrollaron habilidades para la competencia entre equipos de juego; resaltando que, el juego didáctico permite la ruptura de esquemas tradicionales de enseñanza y, facilita a los educandos crear su propia estrategia de cómo resolver un ejercicio y su relación con los aspectos conceptuales [10].

Acerca del uso de videojuegos en el proceso de enseñanza-aprendizaje de la estequiometría no hay aún suficiente estudio, sin embargo, si se ha aplicado 
en la enseñanza de la química en general; es el caso de Abella y García, quienes encontraron que el estudiante profundiza en la naturaleza discontinua de la materia, se divierte y se favorece la comunicación, debido a que en los juegos los estudiantes adquieren un papel activo y son actores principales de su aprendizaje. Como valor agregado, se podrían incluir videojuegos que motiven la comprensión de la naturaleza discontinua de la materia, así como las relaciones matemáticas que prevalecen en sus transformaciones [11].

Los videojuegos como herramienta didáctica aportan beneficios de motivación a los estudiantes y les permite afianzar su autonomía en ambientes educativos, además, fortalecen la autoconfianza y la autoestima, posibilitan el trabajo colaborativo y fomentan la creatividad [12]. En este artículo se plantea que incluir un videojuego como instrumento de apoyo, estimula la resolución de problemas y el mejoramiento de habilidades motoras y cognitivas [13], éstas coinciden con las estrategias que pueden establecerse para superar dificultades detectadas en el proceso de enseñanza y sobretodo del aprendizaje significativo de la estequiometria.

\section{METODOLOGÍA}

La finalidad de esta investigación es determinar si al incluir un videojuego serio como herramienta didáctica de apoyo, se motiva el aprendizaje de los cálculos estequiométricos, se acudió a un método cuantitativo, de enfoque sintético y diseño cuasi experimental [14].

\subsection{Población}

El estudio se aplicó a un total de 28 estudiantes matriculados de la Institución Educativa José María Silva Salazar del municipio de Buenavista, Boyacá, se realizó un muestreo por conveniencia.

\subsection{Recolección de datos}

Los test de diagnóstico y de Bartle propuesto en 2005, así como los Pre-test y Post-test se aplicaron virtualmente dada la situación suscitada por la pandemia por SARS-CoV-2 (coronavirus), así como la aplicación del instrumento, el videojuego serio "Mol2All".

\subsection{Procedimiento}

FASE 1. Caracterización del nivel de interpretación de ecuaciones químicas por parte de los estudiantes. Se aplicó un test para recolectar información sobre los datos generales de los estudiantes de cuatro instituciones educativas (IE) ubicadas en diferentes zonas del departamento de Boyacá: Institución Educativa José María Silva Salazar (IEJMSS), Institución Educativa Técnica Industrial Gustavo Jiménez (IETIGJ), Institución Educativa Valentín García (IEVG) y el Colegio de la Presentación (COLPRE). En este mismo instrumento, las preguntas fueron orientadas a evidenciar cómo perciben los estudiantes su resolución de problemas y las dificultades en la interpretación de ecuaciones químicas basadas en el trabajo de Martínez, M. y De Longhi, A. [4]. Este test diagnostico se elaboró y aplicó empleando Google Forms.

FASE 2. Diseño de la estrategia didáctica basada en los niveles de caracterización. De acuerdo al diagnóstico, se elaboró una estrategia didáctica para facilitar la resolución de problemas estequiométricos, mediada por la intervención del videojuego "Mol2All" como herramienta de apoyo.

FASE 3. Identificación de requisitos del videojuego. Se tuvieron en cuenta dos aspectos: el perfil del jugador, el cual se determinó mediante la metodología de Bartle; y el segundo, la determinación de la accesibilidad y usabilidad para las condiciones técnicas que se acomoden a la población.

\section{RESULTADOS}

FASE 1. Caracterización del nivel de interpretación de ecuaciones químicas por parte de los estudiantes

En la Tabla 1, se recopila la información obtenida al caracterizar cuatro muestras, de diferentes instituciones educativas (IE): Institución Educativa José María Silva Salazar (IEJMSS), Institución Educativa Técnica Industrial Gustavo Jiménez (IETIGJ), Institución Educativa Valentín García (IEVG) y el Colegio de la Presentación (COLPRE), 
permitiendo establecer similitudes y diferencias entre los estudiantes pertenecientes a estos establecimientos.

Tabla 1. Caracterización de las muestras analizadas.

\begin{tabular}{|c|c|c|c|c|}
\hline & IEJMSS & IETIGJ & IEVG & COLPRE \\
\hline Oficial/Privado & Oficial & Oficial & Oficial & Privado \\
\hline $\begin{array}{l}\text { Municipio en el } \\
\text { que se ubica }\end{array}$ & $\begin{array}{l}\text { Buenavista } \\
\text { (occidente) }\end{array}$ & $\begin{array}{l}\text { Sogamoso } \\
\text { (Centro } \\
\text { oriente) } \\
\end{array}$ & $\begin{array}{l}\text { Labranzagrande } \\
\text { (oriente) }\end{array}$ & $\begin{array}{l}\text { Tunja } \\
\text { (Centro) }\end{array}$ \\
\hline $\begin{array}{l}\text { Tamaño de } \\
\text { muestra }\end{array}$ & $\begin{array}{c}14 \\
\text { estudiantes }\end{array}$ & $\begin{array}{c}14 \\
\text { estudiantes }\end{array}$ & $\begin{array}{c}14 \\
\text { estudiantes }\end{array}$ & $\begin{array}{c}14 \\
\text { estudiantes }\end{array}$ \\
\hline $\begin{array}{l}\text { Zona de } \\
\text { residencia }\end{array}$ & $\begin{array}{l}\text { Rural } \\
(80 \%)\end{array}$ & $\begin{array}{l}\text { Urbana } \\
(71,4 \%)\end{array}$ & $\begin{array}{l}\text { Urbana } \\
(81 \%)\end{array}$ & $\begin{array}{l}\text { Urbana } \\
(90 \%)\end{array}$ \\
\hline $\begin{array}{c}\text { Estrato } \\
\text { socioeconómico }\end{array}$ & 1 y 2 & 1 y 2 & 1 y 2 & 4 \\
\hline Edad & $\begin{array}{l}16 \text { años o } \\
\text { más (80\%) }\end{array}$ & $\begin{array}{c}16 \text { años o más } \\
(100 \%)\end{array}$ & $\begin{array}{c}16 \text { años o más } \\
(94 \%)\end{array}$ & $\begin{array}{c}15 \text { años } \\
(62 \%)\end{array}$ \\
\hline Género & $\begin{array}{c}\text { Masculino } \\
(55 \%)\end{array}$ & $\begin{array}{c}\text { Masculino } \\
(57 \%)\end{array}$ & $\begin{array}{c}\text { Femenino } \\
(62 \%)\end{array}$ & $\begin{array}{c}\begin{array}{c}\text { Femenino } \\
(86 \%)\end{array} \\
\end{array}$ \\
\hline $\begin{array}{l}\text { Nivel de } \\
\text { interpretación } \\
\text { de ecuaciones } \\
\text { químicas }\end{array}$ & 2,7 & 3,5 & 2,5 & 2,8 \\
\hline $\begin{array}{l}\text { Autoevaluación } \\
\text { Resolución de } \\
\text { Problemas }\end{array}$ & 3 & 3 & 3,3 & 3,5 \\
\hline
\end{tabular}

Los valores asignados para los ítems de "Nivel de interpretación de ecuaciones químicas" y "Autoevaluación Resolución de Problemas", están en una escala de 0 a 5, siendo 0 el valor más bajo y 5 el más alto. En la Tabla 1, se especifica el valor promedio que tuvieron las muestras en cada uno de los aspectos ya mencionados.

En cuanto al nivel de interpretación de ecuaciones químicas que manejan los estudiantes de las IE estudiadas, se observaron algunas diferencias. Por tal razón, se hizo necesario realizar el análisis comparativo por medio de una prueba T-Student para muestras independientes, empleando la herramienta JASP 0.14.1. Las hipótesis que se pusieron a prueba se basaron en la existencia o no de una diferencia significativa entre las medias del nivel de interpretación de ecuaciones químicas de cada IE. La tabla 2, ilustra mejor estos hallazgos.

Tabla 2. Datos Prueba T-Student para muestras independientes.

\begin{tabular}{|c|c|c|c|c|c|c|}
\hline Comparación & $t$ & df & p & $\begin{array}{l}\text { d de } \\
\text { Cohen }\end{array}$ & $\begin{array}{c}\text { Test de } \\
\text { normalidad }\end{array}$ & $\begin{array}{c}\text { Igualdad de } \\
\text { varianzas }\end{array}$ \\
\hline $\begin{array}{c}\text { IEJMSS } \\
\text { vs. IETIGJ }\end{array}$ & $\begin{array}{l}1,9 \\
18 \\
\end{array}$ & 26 & 0,066 & 0,725 & $\begin{array}{c}0,369 \text { y } 0,339 \\
\text { respectivamente }\end{array}$ & $p=0,572$ \\
\hline $\begin{array}{l}\text { IEJMSS } \\
\text { vs. IEVG }\end{array}$ & $\begin{array}{l}0,4 \\
21 \\
\end{array}$ & 26 & 0,677 & 0,159 & $\begin{array}{l}0,369 \text { y } 0,083 \\
\text { respectivamente }\end{array}$ & $p=0,858$ \\
\hline $\begin{array}{l}\text { IEJMSS } \\
\text { vs. COLPRE }\end{array}$ & $\begin{array}{l}0,2 \\
99\end{array}$ & 26 & 0,767 & 0,113 & $\begin{array}{l}0,369 \text { y } 0,214 \\
\text { respectivamente }\end{array}$ & $p=0,924$ \\
\hline
\end{tabular}

A partir de lo anterior, resultados obtenidos y organizados en la Tabla 2, puede concluirse lo siguiente:

Para el valor de $t$ : las medias de las IE en el nivel de interpretación de ecuaciones químicas no difieren de manera significativa. Para el caso de la comparación entre las muestras IEJMSS y IETIGJ, aunque el valor de t alcanza un valor cercano a $2(1,918)$, no es suficiente para inferir que exista una diferencia marcada entre las medias.

El valor de significancia (valor de $p$ ): para las tres comparaciones, el valor alcanzado por p es mayor a 0,05. Lo cual indica que NO hay una diferencia significativa entre las medias de las IE en cuanto al nivel de interpretación de ecuaciones químicas.

d de Cohen: la comparación entre las muestras IEJMSS y IETIGJ muestra una d igual a 0,725, que demuestra que la diferencia entre las medias está en un nivel medio. En los otros dos casos muestra que la diferencia entre las medias es baja, es decir, tienden a ser similares.

Test de normalidad: los valores tabulados son mayores a 0,05, indicando que la distribución de los datos en las tres comparaciones es uniforme.

Igualdad de varianzas: el valor que alcanzó p en cada caso es mayor a 0,05, lo que se traduce en que existe una igualdad entre las varianzas de las muestras analizadas en las tres comparaciones.

FASE 2. Diseño de la estrategia didáctica basada en los niveles de caracterización

De acuerdo a lo obtenido en la fase 1 de caracterización, en la cual no se encontró diferencia significativa entre los niveles de interpretación de ecuaciones químicas de los cuatro establecimientos educativos analizados, se determinó cuáles son las herramientas que a los estudiantes les son más útiles para resolver con éxito un problema en general: 


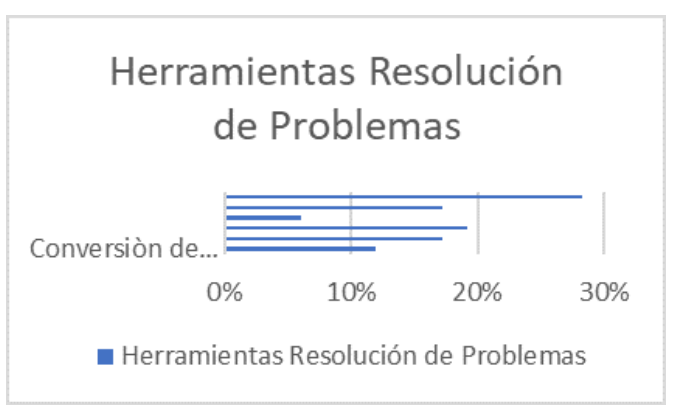

Figura 1. Herramientas empleadas por los estudiantes para la Resolución de Problemas.

Como se observa en la gráfica global, no hay una herramienta que tenga una aceptación del 50\% o más. Las más significativas fueron las siguientes: revisar un ejercicio resuelto paso por paso (28,3\%), establecer la razón molar (19,2\%) y pedir explicación del docente $(17,2 \%)$. Con estas herramientas se elaboraron dos Guías de Aprendizaje, recopilando información de algunos sitios Web y libros de texto especializados, para aplicar en los estudiantes en el modelo de Educación en Casa.

En cuanto a la herramienta "Balance de ecuación", la cual tuvo una aceptación del 17,2\% global de las muestras analizadas, reafirma el bajo nivel de interpretación de ecuaciones químicas que tienen los estudiantes objeto de estudio.

FASE 3. Identificación de requisitos del videojuego Se adaptó el test de Bartle, de acuerdo a las condiciones de la población y éste se aplicó los estudiantes de la IEJMSS para determinar el perfil de jugador de cada uno de ellos. El test arrojó la siguiente información:

Tabla 3. Perfil del jugador según Bartle (2005), Adaptado de Vargas et al. (2019)

\begin{tabular}{clll}
\hline $\begin{array}{c}\text { Perfil } \\
\text { jugador }\end{array}$ & Características & $\begin{array}{c}\text { Aspectos que } \\
\text { llaman su } \\
\text { atención }\end{array}$ & $\begin{array}{c}\text { Perfil } \\
\text { jugador en } \\
\text { la muestra }\end{array}$ \\
\hline & $\begin{array}{l}\text {-Busca ser el } \\
\text { ganador y primero } \\
\text { en la tabla de }\end{array}$ & $\begin{array}{l}\text {-Tabla de } \\
\text { clasificación }\end{array}$ & \\
Competitivo & $\begin{array}{l}\text {-Quicación. } \\
\text {-Quiere ganar a } \\
\text { toda costa. } \\
\text {-Le atrae la } \\
\text { popularidad }\end{array}$ & $\begin{array}{l}\text {-Ranking } \\
\text {-Avance en los }\end{array}$ & \\
& niveles & \\
\hline
\end{tabular}

\begin{tabular}{|c|c|c|c|}
\hline Logrador & $\begin{array}{l}\text {-Busca una } \\
\text { recompensa por } \\
\text { lo que resuelve } \\
\text { retos con agilidad. } \\
\text {-Explora nuevos } \\
\text { niveles y } \\
\text { escenarios. } \\
\text {-Busca la } \\
\text { satisfacción } \\
\text { personal. }\end{array}$ & $\begin{array}{l}\text { Superación de } \\
\text { retos/logros }\end{array}$ & $14,3 \%$ \\
\hline Socializador & $\begin{array}{l}\text {-El juego lo } \\
\text { emplean para } \\
\text { forjar nuevas } \\
\text { amistades } \\
\text {-Les gusta generar } \\
\text { acciones grupales } \\
\text { para que todos } \\
\text { puedan superar } \\
\text { los niveles. } \\
\text {-Olvidan } \\
\text { sus propias } \\
\text { estrategias por } \\
\text { socializar. }\end{array}$ & $\begin{array}{l}\text {-Chats } \\
\text {-Perfiles de } \\
\text { usuarios } \\
\text {-Feedback en } \\
\text { cada nivel }\end{array}$ & $21,4 \%$ \\
\hline Explorador & $\begin{array}{l}\text {-Siempre está en } \\
\text { la autosuperación. } \\
\text {-Se siente atraído } \\
\text { por encontrar } \\
\text { características } \\
\text { ocultas del iuego. }\end{array}$ & $\begin{array}{l}\text {-Diferentes } \\
\text { niveles } \\
\text {-Retos/Logros }\end{array}$ & $57,2 \%$ \\
\hline
\end{tabular}

De acuerdo a estos datos se dedujo que el videojuego requería tener algo de todos los aspectos que llaman la atención a los diferentes perfiles de jugadores y así, que este instrumento pueda ser usado en diferentes contextos educativos, no sólo en el analizado en este estudio.

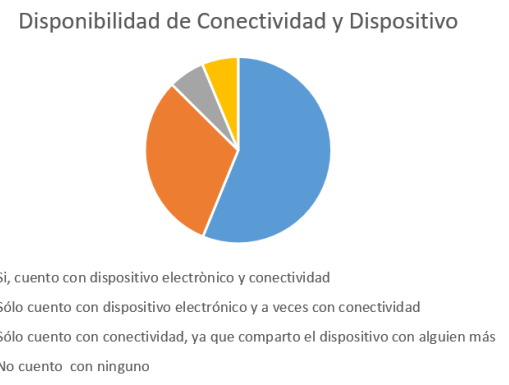

Figura 2. Estudiantes con acceso a conectividad y dispositivo electrónico

La Figura 2 muestra la disponibilidad de conectividad, así como de algún dispositivo electrónico (móvil, Tablet o computador) en los estudiantes de la IEJMSS, con el fin de evaluar, además de los aspectos motivantes para cada perfil de jugador, las características como software que debía tener "Mol2All". Se observa que el 56\% de la muestra analizada tiene acceso a conectividad y algún dispositivo electrónico, mientras que el 31,3\% afirma que 
posee el dispositivo, pero tiene inconvenientes con la conectividad. Con este $87.3 \%$ de la población en cuestión, se tomó la decisión del que el videojuego "Mol2All" fuera liviano, no generara el consumo importante de datos y sea responsivo para poder interactuar con éste, bien sea desde un dispositivo móvil, Tablet o computador, a través de un browser.

\section{CONCLUSIONES}

Con respecto al análisis diagnóstico elaborado a las cuatro muestras de las cuatro diferentes IE evaluadas, pudo determinarse que la mayoría de los estudiantes de educación media, independiente de su zona de residencia, su estrato socioeconómico, si la Institución Educativa es de carácter oficial o privado, presentan deficiencias en la interpretación de las ecuaciones químicas. Todo ello coincide con la premisa de que una de las causas de las deficiencias de la resolución de los problemas radica en la poca apropiación de los conceptos previos, como es el interpretar una ecuación química y reconocer si está balanceada [4].

No existe consenso dentro de los estudiantes de cuál es la mejor herramienta para comprender la forma de realizar los cálculos químicos, sin embargo, el factor motivacional puede ayudar para que se incremente el interés; por este motivo, se estableció que una estrategia didáctica mediada por un videojuego como Mol2All se puede llegar a desarrollar en los estudiantes sus habilidades cognitivas relativas a la resolución de problemas [10] y [11].

El esquema del videojuego Mol2All se deberá estructurar a través de la estrategia didáctica, producto de la recopilación de información y la generación propia de problemas estequiométricos ambientados en la situación actual de pandemia a causa del SARS-CoV-2. Esto con el fin de generar un aprendizaje significativo, puesto que es contexto cotidiano en el que el estudiante asume un papel activo [1], [8] y [9].

El videojuego Mol2All se diseñará para promover las habilidades cognitivas y las relacionadas con la solución de problemas, basado en aspectos que se acerquen a la realidad del estudiante y se ajuste a sus preferencias y necesidades formativas [13]. La recolección de información sobre la disponibilidad en conectividad y dispositivo electrónico, así como determinar el perfil de jugador de nuestros estudiantes (metodología de Richard Bartle), permitirá direccionar el diseño de Mol2All para que cumpla con la mayoría de las expectativas de la muestra analizada, además de alcanzar su objetivo no sólo de entretenimiento, sino también de potenciación de habilidades relativas a la resolución de problemas [15].

\section{REFERENCIAS}

[1] S. M. Obando, Implementación de estrategias didácticas para la enseñanza de la estequiometría en estudiantes del grado once de enseñanza media, Medellín: Repositorio Universidad Nacional, 2013.

[2] «Estequiometría y ley de conservación de la masa: lo que puede ocultar la simplificación del discurso experto,» Ciênc. Educ., Bauru, vol. 21, no 2, pp. 351360, 2015.

[3] C. D. Bridges, Experiences Teaching Stoichiometry to Students in Grades 10 and 11, Washington D.C.: Walden Dissertations and Doctoral Studies Collection, 2015, p. 145.

[4] M. S. Martínez y A. L. De Longhi, «Identificación y categorización de dificultades de lectocomprensión en enunciados de lápiz y papel de estequiometría,» Revista Eureka de Enseñanza y divulgación de las ciencias, vol. 10, no 2, pp. 159-170, 2013.

[5] G. E. Hurtado, "Las estrategias didácticas activas en el aprendizaje de la resolución de problemas de química, influencia del estilo cognitivo del estudiante,» Tecné, Episteme y Didaxis: TED, vol. 39, no 1, pp. 31-51, 2016.

[6] M. O. Castelán y G. Hernández, Estrategia didáctica para apoyar la comprensión de la estequiometría a partir del uso de analogías, Veracruz: X Congreso nacional de investigación educativa COMIE, 2009.

[7] A. Raviolo y G. Lerzo, «Enseñanza de la estequiometría: uso de analogías y comprensión 
conceptual,»Educación Química, vol. 27, no1, pp. 195-204, 2016.

[8] A. Raviolo y G. Lerzo, «Analogías en la enseñanza de la estequiometría: revisión de páginas web,» Revista electrónica de investigación en educación en ciencias, vol. 9, no 2, pp. 28-41, 2014.

[9] D. A. Mojica, Ambiente de aprendizaje virtual como apoyo para la enseñanza de estequiometría en grado décimo, Bogotá: Repositorio Universidad Nacional, 2013.

[10] K. Marcano, «Aplicación de un juego didáctico como estrategia pedagógica para la enseñanza de la estequiometría, " Revista de Investigación No.84, vol. 39, no 1, pp. 181-204, 2015.

[11] L. Abella y Á. García, «El uso de videojuegos para la enseñanza de las ciencias, nuevos desafíos al papel docente,» EDUCyT, vol. 84, no 2, pp. 19-32, 2014.

[12] F. Quintanal, «Aplicación de minijuegos en física y química de bachillerato,» Historia y Comunicación Social, vol. 18, no No. Especial Octubre, pp. 411420, 2013.

[13] A. P. Roncancio, M. F. Ortiz, H. Llano, M. J. Malpica y J. J. Bocanegra, «El uso de los videojuegos como herramienta didáctica para mejorar la enseñanzaaprendizaje: una revisión del estado del tema,» Revista Ingeniería, Investigación y Desarrollo, vol. 17, ํo 2, pp. 36-46, 2017.

[14] R. Hernández Sampieri, C. Fernández y P. Baptista, Metodología de la investigación, Quinta Edición, México D.F.: Mc Graw Hill, 2010.

[15] Z. Vargas, A. Rodríguez y M. Mendoza, «Modelo de integración de gamificación como estrategia de aprendizaje para colegios virtuales. Caso: Sogamoso-Colombia,» Espacios, vol. 40, no 12, pp. 12-26, 2019.

[16] A. Raviolo, «Simulando estequiometría con la hoja de cálculo: uso de la barra de desplazamiento,» Journal of Science Education, vol. 18, no 1, pp. 3034, 2017. 